\title{
Epidermal growth factor receptor kinase substrate 8 promotes the metastasis of cervical cancer via the epithelial-mesenchymal transition
}

\author{
QIAN LI, WEI BAO, QIONG FAN, WEN-JING SHI, ZHU-NAN LI, YING XU and DAN WU
}

Department of Obstetrics and Gynecology, International Peace Maternity and Child Health Hospital of China Welfare Institute, Shanghai Jiao Tong University School of Medicine, Shanghai 200030, P.R. China

Received July 29, 2015; Accepted July 27, 2016

DOI: $10.3892 / \mathrm{mmr} .2016 .5638$

\begin{abstract}
Epidermal growth factor receptor pathway substrate 8 (Eps8) has been identified as a novel substrate for epidermal growth factor receptor (EGFR) kinase and is involved in EGFR-mediated signaling pathways correlated with tumorigenesis, proliferation and metastasis in various cancer types. However, the precise role of Eps8 in cervical cancer metastasis remains to be elucidated. Immunohistochemistry revealed that Eps8 was significantly increased in cervical cancer specimens compared with squamous intraepithelial lesion and normal cervical tissues. Additionally, it was revealed that Eps8 expression not only correlated with cervical cancer progression, but also exhibited a close correlation with the epithelial-mesenchymal transition (EMT) markers, E-cadherin and vimentin. Furthermore, the present study focused predominantly on the EMT-associated role of Eps8 in the EMT, migration and invasion of cervical cancer cells. Eps8-short hairpin (sh) RNA was transfected into HeLa and SiHa cells to deplete its expression, and reverse transcription-quantitative polymerase chain reaction and western blot analyses were performed to confirm Eps8-knockdown and to investigate the influence of Eps8 on EMT markers. The present findings have revealed that Eps8 silencing led to the upregulation of the epithelial marker E-cadherin, while expression of the mesenchymal marker vimentin and the transcription factor snail was decreased at both mRNA and protein expression levels. Transwell cell migration and Matrigel invasion assays showed that downregulation of Eps8 significantly inhibited cell migration and invasion of HeLa and $\mathrm{SiHa}$ cells. Taken together, these results
\end{abstract}

Correspondence to: Dr Dan Wu, Department of Obstetrics and Gynecology, International Peace Maternity and Child Health Hospital of China Welfare Institute, Shanghai Jiao Tong University School of Medicine, 910 Hengshan Road, Shanghai 200030, P.R. China

E-mail: dr.wudan@163.com

Key words: Eps8, cervical cancer, cervical squamous intraepithelial lesion, epithelial-mesenchymal transition, metastasis suggested that Eps8 promotes cervical cancer metastasis by orchestrating the EMT.

\section{Introduction}

Despite the great progress that has been made in early screening of cervical cancer programs, cervical cancer remains the leading cause of gynecological cancer-associated mortalities worldwide (1). Persistent high-risk human papilloma virus infection is suggested to be the prerequisite for cervical cancer (2); however, the virus alone is insufficient for the development of cervical cancer, suggesting that other molecular events are involved in the initiation and progression of cervical cancer (3). The expression of molecules associated with tumor invasion and metastasis indicate an unfavorable prognosis (4). The present understanding of the molecular mechanisms contributing to the invasion-metastasis of cervical cancer remains limited, although accumulating evidence suggests that the epithelial-mesenchymal transition (EMT) serves a pivotal role in the initial step of tumor invasion-metastasis (5). Consequently, the EMT has become the focus of cervical cancer metastasis research.

The EMT is a process of switching the epithelial cell phenotype to a more loosely fibroblast-like phenotype (6). This is coupled with the loss of cell polarity and intercellular junctions, remodeling of the cytoskeleton and alteration of various EMT-related markers, including downregulation of epithelial markers, including E-cadherin, and upregulation of the mesenchymal markers $\mathrm{N}$-cadherin and vimentin (6). These changes provide cancer cells with elevated invasive/metastatic capacities and therapeutic resistance (7).

Epidermal growth factor receptor pathway substrate 8 (Eps8) was initially identified as a novel substrate for epidermal growth factor receptor (EGFR) kinase (8). Overexpression of Eps8 facilitates enhanced mitogenesis and malignant transduction via EGFR-mediated activation of downstream signaling pathways (9). Previous studies have revealed that Eps8 is highly expressed in various human tumor types, including colorectal, pituitary, oral, esophageal, pancreatic, ovarian, lung, breast, thyroid and cervical cancer (10-17). Eps8 overexpression has also been shown to be involved in numerous signaling pathways associated with 
tumorigenesis, metastasis and proliferation of cancer, and represents an unfavorable prognostic biomarker in patients with cancer $(10,12-14,17)$.

Epithelial growth factor (EGF) is suggested to be one of the most potent EMT regulatory factors in cervical cancer. Overexpression of EGFR in cervical cancer indicates poor clinical prognosis and EGF-mediated EMT may account for this (18-20). More recent studies have shown that chemoresistance is associated with the EMT in cancer cells, and the acquired chemotherapeutic resistance is often accompanied by the transformation from the epithelial to the mesenchymal phenotype in cancer cells (21-23). Chen et al (17) found that Eps8 knockdown contributes to increased sensitivity to chemotherapy. Based on these findings, it was hypothesized that Eps8 is associated with the process of EMT in cervical cancer.

In the present study, immunohistochemistry was used to detect the expression of Eps8 in different cervical samples. Correlations between Eps8 expression and the clinicopathological characteristics, as well as EMT associated proteins in cervical cancer tissues were further analyzed. RNA interference (RNAi) was applied to inhibit the expression of Eps8 in cervical cancer cells, reverse transcription-quantitative polymerase chain reaction (RT-qPCR) and western blot analyses were performed to investigate the influence of Eps8 knockdown on EMT markers at the mRNA and protein expression levels. Transwell cell migration and Matrigel invasion assays were used to investigate the effects of Eps8-knockdown on the capacity of invasive and migration in HeLa and SiHa cells.

\section{Materials and methods}

Cell culture and clinical specimens. Human HeLa and $\mathrm{SiHa}$ cervical cancer cell lines were purchased from the Cell Bank of China (Shanghai, China) and were maintained in Dulbecco's modified Eagle's medium (DMEM; Gibco; Thermo Fisher Scientific, Inc., Waltham, MA, USA) supplemented with $10 \%$ fetal bovine serum (FBS; Gibco; Thermo Fisher Scientific, Inc.) and $1 \%$ penicillin/streptomycin solution (Gibco; Thermo Fisher Scientific, Inc.). The cells were cultured at $37^{\circ} \mathrm{C}$ in a humidified atmosphere containing $5 \% \mathrm{CO}_{2}$.

A total of 134 paraffin-embedded cervical specimens, including 16 normal cervical epithelia (NC), 25 low-grade squamous intraepithelial lesion (LSIL), 30 high-grade squamous intraepithelial lesion (HSIL) and 63 squamous cervical cancer (SCC) specimens, were obtained from the Pathology Department at the International Peace Maternity and Child Health Hospital of the China Welfare Institute (Shanghai, China) between 2008 and 2014. The age of the patients was between 29 and 68 years (mean age, 42 years). The NC specimens were obtained from patients who underwent hysterectomies for uterine fibroids, the LSIL and HSIL specimens were obtained from patients who underwent cervical conization. None of the patients enrolled in the present study received preoperative chemotherapy, radiotherapy or immunotherapy. The tumor clinical stages and clinicopathological classifications were based on the International Federation of Gynecology and Obstetrics (FIGO) criteria. Approval for the use of these specimens was granted by the Ethics Committee of the International Peace Maternity and Child Health Hospital of the China Welfare Institute. The main clinical characteristics of the patients with cervical cancer are summarized in Table I.

Immunohistochemical analysis. Immunohistochemical staining was performed on $4 \mu \mathrm{m}$ thick paraffin-embedded tissue sample sections. Briefly, the sections were dewaxed with xylene twice for $15 \mathrm{~min}$ and rehydrated through a graded alcohol series $(100,90$ and $70 \%)$ at room temperature. Antigen retrieval was performed using citrate buffer $\left(\mathrm{pH} \mathrm{6.0)}\right.$ at $95^{\circ} \mathrm{C}$ for $15 \mathrm{~min}$ and endogenous peroxidase activity was quenched by immersion in $3 \% \mathrm{H}_{2} \mathrm{O}_{2}$ for $10 \mathrm{~min}$ at room temperature. Subsequently, tissue sections were blocked by incubation in $1 \%$ bovine serum albumin (BSA; Beyotime Institute of Biotechnology, Beijing, China) for $30 \mathrm{~min}$ at room temperature to prevent non-specific binding. The tissue sections were then incubated at $4^{\circ} \mathrm{C}$ overnight with anti-Eps8 (1:200; ab203272; Abcam, Cambridge, UK), anti-E-cadherin (1:300; 3195S; Cell Signaling Technology, Inc., Danvers, MA, USA) and anti-vimentin (1:200; 5741S; Cell Signaling Technology, Inc.) primary rabbit monoclonal antibodies. Following incubation with the primary antibodies, the tissues sections were incubated at $37^{\circ} \mathrm{C}$ for $30 \mathrm{~min}$ with horseradish peroxidase-conjugated goat anti-rabbit secondary antibody (1:500; GTX26721; GeneTex Inc., Irvine, CA, USA). The 3,3'-diaminobenzidine substrate was applied to visualize the immunoreactivity prior to counterstaining the slides with $10 \%$ hematoxylin, dehydrating in graded ethanol and cleared in xylene. For negative controls, phosphate-buffered saline was substituted for the primary antibodies. The expression levels were evaluated by two pathologists in a blinded manner. The protein expression levels of Eps8, E-cadherin and vimentin were scored semi-quantitatively by multiplying the 'percent positivity score' by the 'staining intensity score'. The percent positivity was scored according to the following criteria: $0,<10 \% ; 1,10-24 \% ; 2,25-50 \% ; 3,>50 \%$ positive tumor cells. Staining intensity was graded as 0 , no staining; 1 , weak staining; 2 , moderate staining; or 3 , strong staining. The final score was defined as follows: 0, negative; 1-4, low expression; 5-12, high expression.

Transient transfection. The short hairpin (sh)RNA sequence targeting Eps8 (Eps8-shRNA: 5'-GCTGTGAGCCTGATT GATTTA-3') and the negative control shRNA were synthesized and cloned into the pGPU6/green fluorescent protein (GFP)/Neo vector (GenePharma, Shanghai, China). The HeLa $\left(2 \times 10^{5}\right)$ or $\mathrm{SiHa}\left(4 \times 10^{5}\right)$ cells were grown in 6 -well plates at $70-80 \%$ confluence prior to transient transfection with a mixture of Lipofectamine 2000 (Invitrogen; Thermo Fisher Scientific, Inc.) and Eps8 shRNA or control shRNA, according to the manufacturer's instructions. Following incubation for $6 \mathrm{~h}$, the transfection medium was replaced with regular medium and after $48 \mathrm{~h}$, GFP expression was evaluated by fluorescent microscopy (Zeiss Axioskop 40; Zeiss, Oberkochen, Germany), for the qualitative assessment of the transfection efficiency. The cells were harvested at $48 \mathrm{~h}$ post-transfection for RNA analyses and at $72 \mathrm{~h}$ post-transfection for protein analyses to confirm the downregulation of Eps8 expression.

RNA extraction and RT-qPCR. The total RNA from HeLa and SiHa cells was extracted using TRIzol reagent (Invitrogen; 


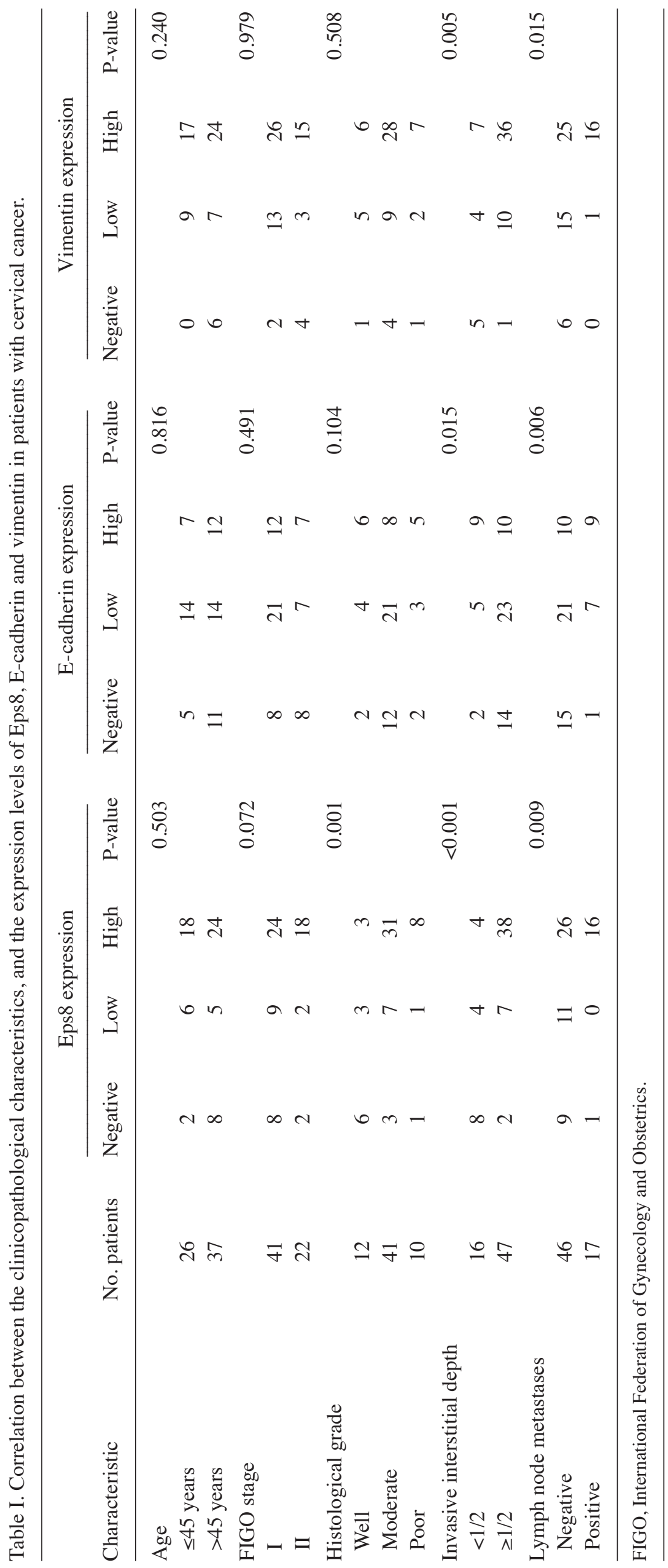


Thermo Fisher Scientific, Inc.). The extracted RNA $(2 \mu \mathrm{g})$ was reverse-transcribed into cDNA using the Prime Script RT reagent kit (Takara Bio, Inc., Dalian, China), according to the manufacturer's protocol. RT-qPCR was then performed in Eps8-silenced and control cells using the cDNA as a template and the SYBR Green PCR kit (Takara Bio, Inc.), according to the manufacturer's protocol. All primers used in this experiments were designed using Premier 5.0 software and their sequences are listed in Table II. The PCR reaction conditions were as follows: $95^{\circ} \mathrm{C}$ for $3 \mathrm{~min}$, followed by 40 cycles at $95^{\circ} \mathrm{C}$ for $10 \mathrm{sec}$ and $60^{\circ} \mathrm{C}$ for $30 \mathrm{sec}$. The target gene expression levels were normalized against that of GAPDH. Quantification of the relative expression of target genes was calculated using the comparative $2^{-\Delta \Delta \mathrm{Cq}}$ method (24). All experiments were performed in triplicate.

Western blot analysis. HeLa and SiHa cells were harvested and washed twice using ice-cold PBS. The cells were subsequently lysed on ice for $30 \mathrm{~min}$ with radioimmunoprecipitation buffer (Beyotime Institute of Biotechnology, Beijing, China), containing $1 \mathrm{mM}$ phenylmethanesulfonyl fluoride. The lysates were subsequently centrifuged at $12,000 \mathrm{x}$ g at $4^{\circ} \mathrm{C}$ for $15 \mathrm{~min}$. The protein concentrations were measured using bicinchoninic acid protein assays (Beyotime Biotechnology). Equivalent quantities $(60 \mu \mathrm{g})$ of total protein were separated on $10 \%$ sodium dodecyl sulfate-polyacrylamide gel electrophoresis gel and blotted onto a polyvinylidene membrane (EMD Millipore, Billerica, MA, USA). Following blocking in 5\% BSA at room temperature for $2 \mathrm{~h}$, the membranes were incubated overnight at $4^{\circ} \mathrm{C}$ with the following primary rabbit monoclonal antibodies: Anti-Eps8 (1:1,000; Abcam), anti-E-cadherin (1:1,000; Cell Signaling Technology, Inc.), anti-vimentin $(1: 1,000$; Cell Signaling Technology, Inc.) and anti-GAPDH (1:2,000; Tiangen Biotech Co., Ltd., Beijing, China). The membranes were washed in Tris-buffered saline containing $0.1 \%$ Tween-20 three times and were subsequently incubated with horseradish peroxidase-conjugated goat-anti-rabbit secondary antibody (1:2,000; GeneTex Inc.) for $1 \mathrm{~h}$ at room temperature. Target protein bands were visualized by electrochemical luminescence using an Enhanced Chemiluminescence Plus kit (Beyotime Institute of Biotechnology), according to the manufacturer's protocol. The levels of target proteins were normalized against that of GAPDH using Image Pro Plus 6.0.

Cell migration and invasion assays. The migration/invasion assay was performed using 24-well Transwell chambers (Corning, Inc., Glendale, AZ, USA) containing polycarbonate filters ( $8 \mu \mathrm{m}$ pore size) that were uncoated/coated with Matrigel (BD Biosciences, Hercules, CA, USA). At $48 \mathrm{~h}$ after transfection, $2 \times 10^{4}$ cells were resuspended in $200 \mu \mathrm{l}$ serum-free DMEM medium with $0.1 \%$ BSA and were added to the upper chambers, while $500 \mu$ 1 DMEM with $20 \%$ FBS was placed in the lower chambers. Following incubation for $24 \mathrm{~h}$, the non-migratory/non-invasive cells on the upper chamber surface were wiped off using a cotton swab and the cells that had migrated/invaded to the lower membrane surface were fixed for $20 \mathrm{~min}$ in $4 \%$ paraformaldehyde, and stained for $15 \mathrm{~min}$ with $0.1 \%$ crystal violet. A total of 10 random visual fields were selected under an inverted microscope (magnification, $\mathrm{x} 200$ ) to capture images and count the
Table II. Quantitative polymerase chain reaction primer sequences.

Gene $\quad$ Sequence (5'-3')

\section{Eps8}

Forward

TGAATGGCTACGGATCATCACC

Reverse: CACTGTCCCGTGCATAATTCT

E-cadherin

Forward

Reverse

CGAGAGCTACACGTTCACGG

Vimentin

Forward GGGTGTCGAGGGAAAAATAGG

Reverse

GACGCCATCAACACCGAGTT

Snail

Forward

Reverse CTTTGTCGTTGGTTAGCTGGT

\section{GAPDH}

Forward

Reverse

TCGGAAGCCTAACTACAGCGA AGATGAGCATTGGCAGCGAG

Esp8, epidermal growth factor receptor kinase substrate 8; GAPDH, glyceraldehyde 3-phosphate dehydrogenase.

number of migrated/invading cells. Each assay was performed in triplicate.

Statistical analysis. Each experiment was performed in triplicate. SPSS 17.0 software was used to perform statistical analysis. The data are presented as the mean \pm standard deviation. Kruskal-Wallis one-way analysis of variance or Student's unpaired t-test was applied to evaluate significant differences. Correlations were assessed using the Pearson's correlation coefficient test. $\mathrm{P}<0.05$ was considered to indicate a statistically significant difference.

\section{Results}

Expression levels of Eps8, E-cadherin and vimentin in the normal cervix, LSIL, HSIL and SCC. To investigate the expression of Eps8 and its association with EMT-related proteins, immunohistochemistry was performed using Eps8, E-cadherin and vimentin antibodies in NC, LSIL, HSIL and SCC (Fig. 1A). Eps8 and vimentin were predominantly localized in the cytoplasm of cervical cancer tissues, and were absent from the 16 normal cervix samples. Weak expression of Eps8 and vimentin was observed in 12/25 (48.0\%) LSIL specimens, and 13/30 (43.3\%) and 15/30 (50.0\%) of the $30 \mathrm{HSIL}$ specimens, respectively. Of the $63 \mathrm{SCC}$ cases, the staining intensity of Eps8 was weak and strong in $11(17.5 \%)$ and $42(66.7 \%)$ specimens, respectively. In addition, vimentin was expressed weakly in 16 cases (25.4\%) and strongly in 41 cases $(65.1 \%)$. E-cadherin was expressed predominantly in the plasma membrane of epithelial cells in the NC. Based on the expression scores, the percentage of high E-cadherin-expressing samples decreased gradually 
A
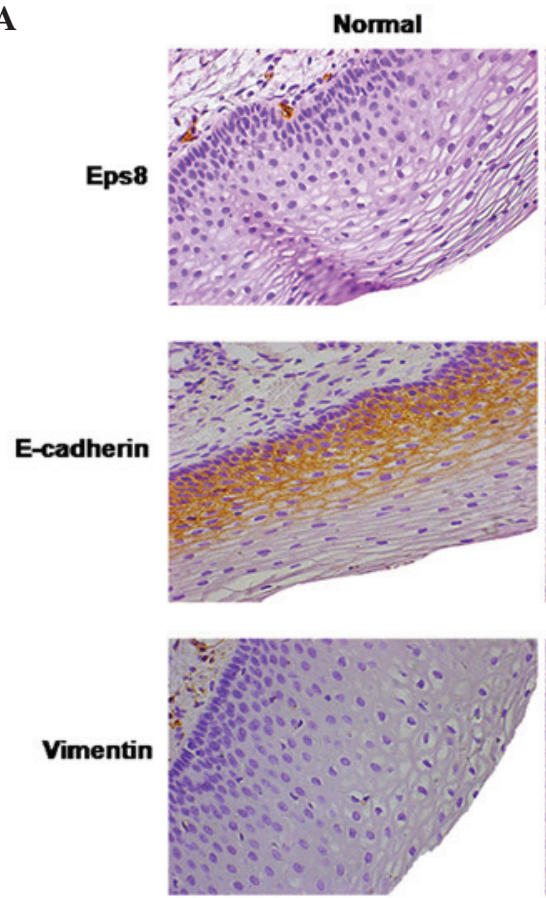

B

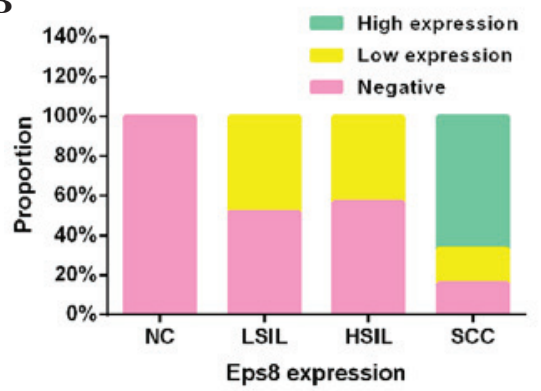

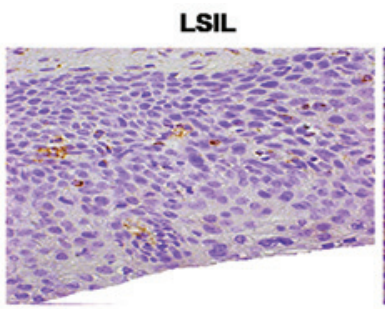
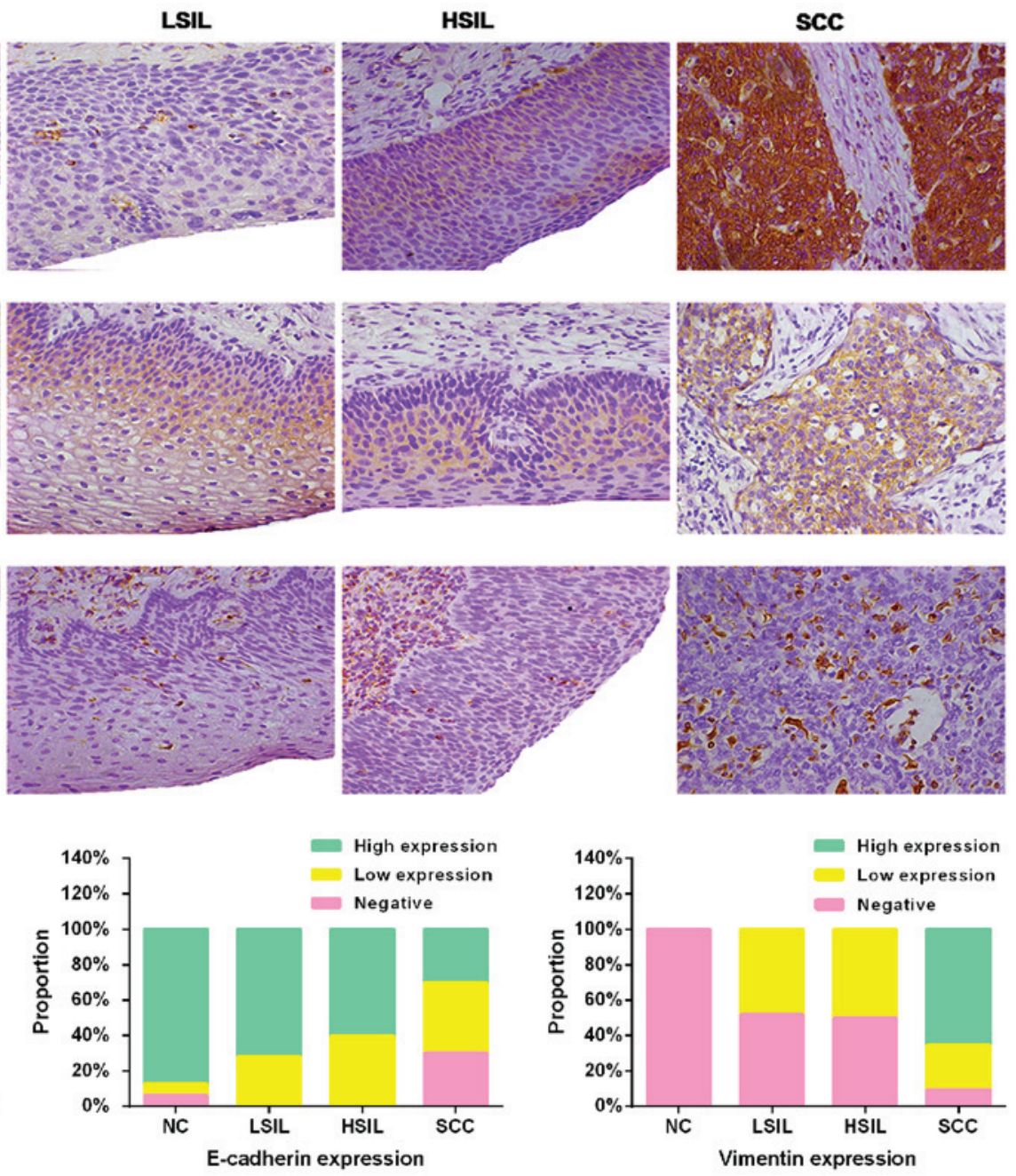

Figure 1. Expression levels of Eps8 and EMT markers in cervical cancer samples. (A) Immunohistochemical staining for Eps8 and the EMT markers, E-cadherin and Vimentin, in NC, LSIL, HSIL and SCC tissues (magnification, x400). (B) The expression levels of Eps8, E-cadherin and Vimentin were categorized as negative, low or high expression in the normal cervix, LSIL, HSIL, and SCC samples. Esp8, epidermal growth factor receptor kinase substrate 8; EMT, epithelial-mesenchymal transition; NC, normal cervical epithelia; LSIL, low-grade squamous intraepithelial lesion; HSIL, high-grade squamous intraepithelial lesion; SCC, squamous cell carcinoma.

from $87.5 \%$ (14/16) in NC, $>72.0 \%(18 / 25)$ in LSIL and $>60.0 \%$ $(18 / 30)$ in HSIL to $30.2 \%(19 / 63)$ in SCC (Fig. 1B).

Association of the expression levels of Eps8, E-cadherin, vimentin with the clinicopathological characteristics of patients with SCC. As shown in Table II, Eps8 expression was significantly associated with histological grade $(\mathrm{P}=0.001)$, invasive interstitial depth $(\mathrm{P}<0.001)$ and lymph node metastases $(\mathrm{P}=0.009)$. However, little association was demonstrated between the expression of Eps8 and age ( $\mathrm{P}=0.503)$, and FIGO stage $(\mathrm{P}=0.072)$. The expression levels of $\mathrm{E}$-cadherin and vimentin demonstrated a marked correlation with invasive interstitial depth $(\mathrm{P}=0.015$ and $\mathrm{P}=0.005$, respectively) and lymph node metastases $(\mathrm{P}=0.006$ and $\mathrm{P}=0.015$, respectively).

Expression of Eps8 correlates with EMT-related proteins in cervical cancer samples. The present study further explored correlations between the expression of Eps8 and EMT marker proteins in 63 samples of cervical SCC tissues. In accordance with the changes in the expression of proteins during the EMT process, the reduced expression of E-cadherin was significantly associated with the increased expression of vimentin $(\mathrm{P}=0.001 ; \mathrm{r}=-0.398)$. Furthermore, a significant negative correlation was observed between the expression levels of E-cadherin and Eps8 ( $\mathrm{P}=0.004 ; \mathrm{r}=-0.355)$, and the expression of Eps8 correlated positively with that of vimentin $(\mathrm{P}<0.001$; $\mathrm{r}=0.562$ ) (Table III). These analyses suggested that Eps8 is associated with the expression of the EMT-related proteins and may be an important indicator of the EMT process in cervical cancer.

Knockdown of Eps8 led to increased expression of epithelial markers and reduced expression of mesenchymal markers. To further characterize the role of Eps8 in the process of the EMT in cervical cancer, Eps8-targeting shRNA and control shRNA plasmids were designed and transiently transfected into HeLa and SiHa cells. To confirm Eps8-knockdown and to investigate the influence of Eps8 on EMT markers, RT-qPCR and western blot analyses were performed in Eps8-inhibited cell lines and control cells. As shown in Fig. 2, Eps8 expression in Eps8-inhibited cell lines was significantly downregulated at both the mRNA and protein 
Table III. Association between the expression levels of Eps8, E-cadherin and vimentin in patients with cervical cancer.

\begin{tabular}{|c|c|c|c|c|c|c|c|c|c|c|}
\hline \multirow[b]{2}{*}{ Definition } & \multicolumn{5}{|c|}{ Eps8 expression } & \multicolumn{5}{|c|}{ Vimentin expression } \\
\hline & Negative & Low & High & P-value & $\mathrm{r}$ & Negative & Low & High & P-value & $\mathrm{r}$ \\
\hline E-cadherin expression & & & & 0.004 & -0.355 & & & & 0.001 & -0.398 \\
\hline Negative & 2 & 1 & 13 & & & 1 & 2 & 13 & & \\
\hline Low & 2 & 3 & 23 & & & 1 & 5 & 22 & & \\
\hline High & 6 & 7 & 6 & & & 4 & 9 & 6 & & \\
\hline Vimentin expression & & & & $<0.001$ & 0.562 & & & & & \\
\hline Negative & 6 & 0 & 0 & & & & & & & \\
\hline Low & 3 & 9 & 4 & & & & & & & \\
\hline High & 1 & 2 & 38 & & & & & & & \\
\hline
\end{tabular}

$\mathbf{A}$

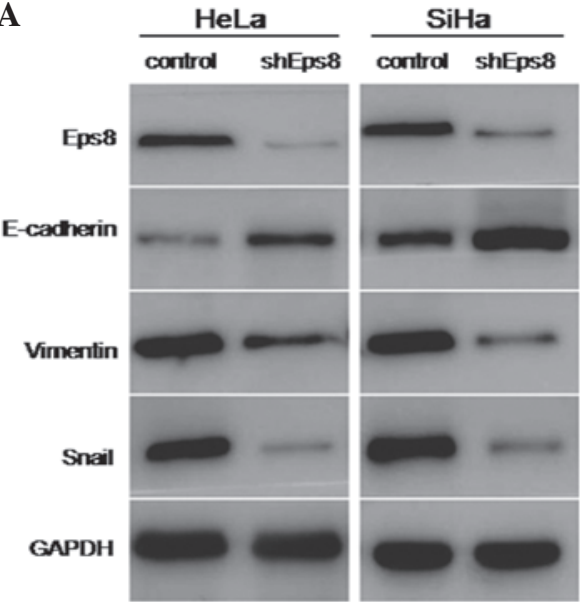

B

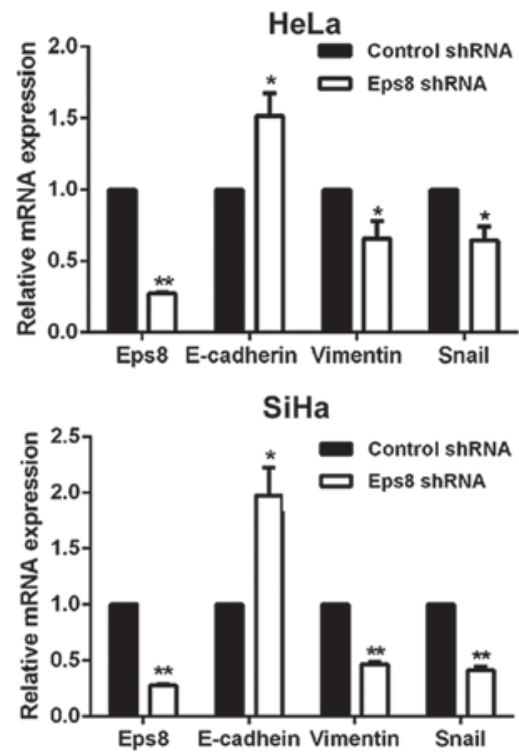

Figure 2. Knockdown of Eps8 influences EMT markers in HeLa and SiHa cells. (A) Western blotting confirmed the upregulation of E-cadherin and downregulation of Vimentin and snail at the protein level in HeLa and $\mathrm{SiHa}$ cells following Eps8 knockdown. GAPDH was used as a loading control. (B) Reverse transcription-quantitative polymerase chain reaction revealed that Eps8 knockdown in HeLa and SiHa cells increased the mRNA expression of E-cadherin, but decreased the mRNA expression levels of vimentin and snail expression. The data are presented as the mean \pm standard deviation of triplicate experiments $\left({ }^{* * *} \mathrm{P}<0.01,{ }^{*} \mathrm{P}<0.05\right.$ compared with the control shRNA). Esp8, epidermal growth factor receptor kinase substrate 8; GAPDH, glyceraldehyde 3-phosphate dehydrogenase; sh, short hairpin. expression levels compared with the control cells. RT-qPCR analysis demonstrated that knockdown of Eps8 led to increased expression of the epithelial marker, E-cadherin, and decreased expression of the mesenchymal marker, vimentin, as well as a key EMT inducer, snail, at the mRNA level (Fig. 2A). Additionally, western blot analysis revealed that the inhibition of Eps8 resulted in downregulated protein expression of vimentin and snail (Fig. 2B). Taken together, these results revealed that Eps8 is involved in EMT progression and that inhibition of Eps 8 impairs the process of the EMT in cervical cancer.

Eps8 silencing represses cell migration and invasion. The present study subsequently performed Transwell cell migration and Matrigel invasion assays to investigate the effects of Eps8 knockdown on the regulation of cell migration and invasion in HeLa and SiHa cells. As shown in Fig. 3, the Transwell migration assay demonstrated that Eps8 knockdown notably inhibited the migration capacity of the Eps8 shRNA transfected cells compared with that of the control cells $(\mathrm{P}<0.01$; Fig. 3$)$. Matrigel invasion assays also demonstrated that transfection with Eps8 shRNA plasmids effectively impaired the invasive capacity of HeLa and SiHa cells $(\mathrm{P}<0.01$; Fig. 3). These results suggested that Eps8 knockdown decreases the migration and invasiveness of HeLa and SiHa cell.

\section{Discussion}

The progression of malignant tumors is a sophisticated process involving multiple factors and molecular events. The EMT, which is believed to be a crucial step in tumor metastasis (25), allows cancer cells to invade adjacent tissues and metastasize to distance sites. It is important to understand the molecular mechanisms underlying the EMT pathway and identification of ways to prevent or reverse the process are critical to the development of novel therapeutic strategies against cancer. Several signaling pathways, including the Wnt, transforming growth factor- $\beta$, EGF, Notch and fibroblast growth factor pathways, have been shown to be involved in regulating the process of the EMT by modulating the expression of certain key transcription factors, including snail, ZEB and twist, which suppress the expression of E-cadherin and activate the mesenchymal transcriptomes $(26,27)$. 
A

HeLa

$\mathrm{SiHa}$
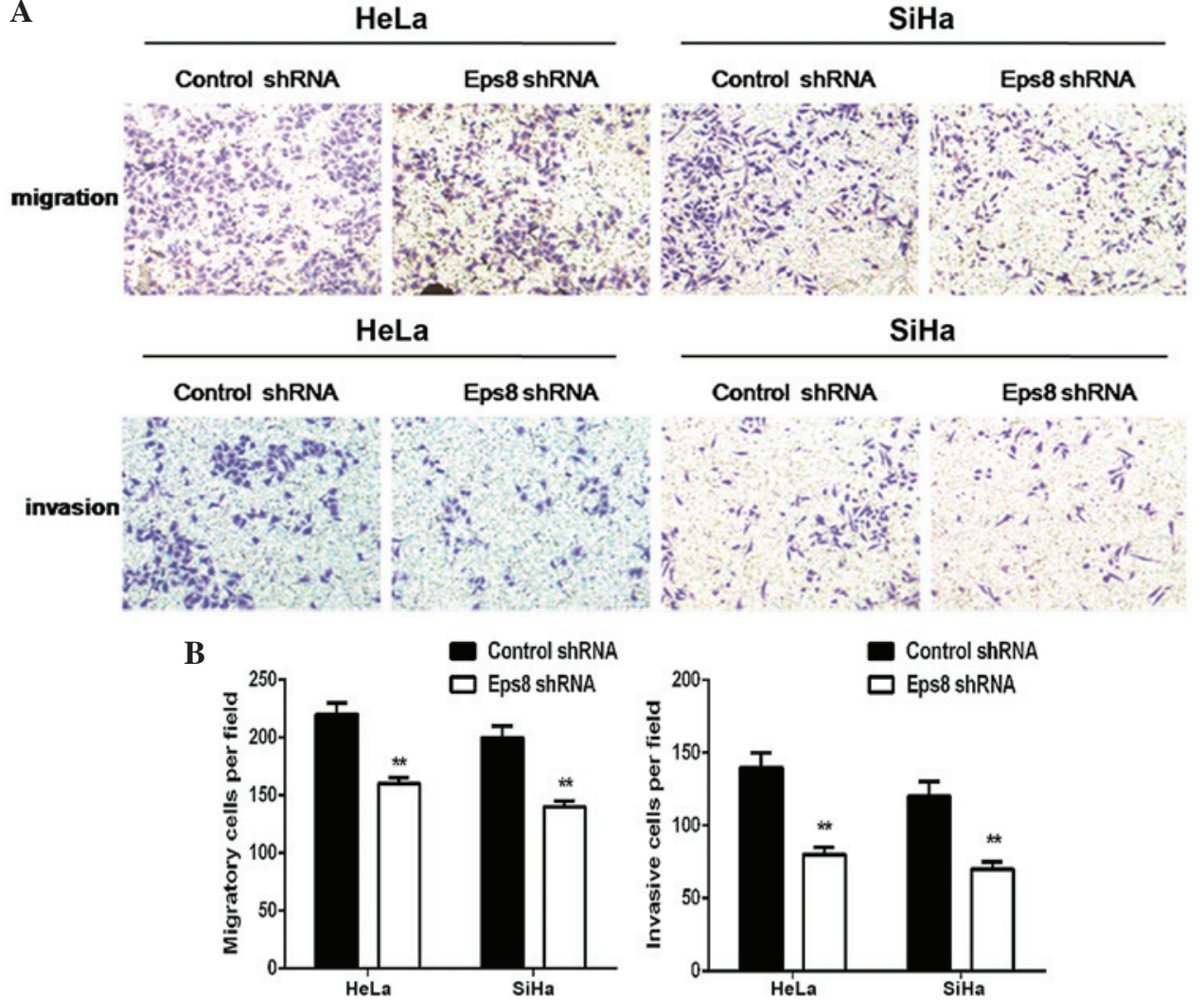

Eps8 shrna

B

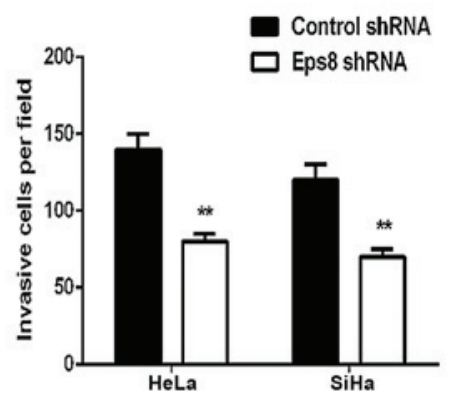

Figure 3. Effects of Eps8 knockdown on the migration and invasion capacity of cervical cancer cells. (A) Transwell migration and invasion assays were performed in Eps8-shRNA transfected HeLa and SiHa cells. Following incubation for $24 \mathrm{~h}$, migrated/invading cells were counted under a microscope and images were captured. Figures are representative of multiple experiments. (B) The data were quantified and are presented as the mean \pm standard deviation of three independent experiments ( ${ }^{* *} \mathrm{P}<0.01$ compared with the control shRNA). Esp8, epidermal growth factor receptor kinase substrate 8; sh, short hairpin.

Eps8 is a novel phosphorylation substrate for EGFR, which enhances the cellular transformation of EGFR-dependent mitogenic signals upon overexpression (28). As an actin-capping protein, the role of Eps8 in cancer metastasis has aroused widespread concern, resulting in a number of studies to elucidate the mechanisms by which Eps8 influences tumor migration. Eps8 overexpression is responsible for enhanced Rac-induced actin cytoskeleton remodeling and cell migration by formation of Eps8-Abi-1-Sos-1 and Eps8-IRSp53 complexes, which are involved in transducing signals to activate the Rho-GTPase family members Rac and Cdc42 (29-31). In addition to the role of Eps8 in tumor metastasis mediated via Rac-dependent pathways, Eps8 is also involved in tumor migration mediated via Rac-independent pathways, including the the Eps8/AKT/FOXM1/matrix metalloproteinase-9 cascade (32). Although considerable efforts have been made to clarify the functions of Eps8 in the metastasis of numerous cancer types, the role of Eps8 in the development of cervical cancer remains to be elucidated. Chen et al (17) analyzed the expression levels of Eps8 in 110 cervical cancer samples by immunohistochemistry and observed that increased Eps8 expression was associated with shorter disease-free survival and overall survival (17). These observations suggested that Eps8 serves a role in the progression of cervical cancer. Therefore, the present study hypothesized that Eps8 is also involved in cervical cancer metastasis. The present study focused predominantly on the EMT-related role of Eps8 in this process.
The present data demonstrated that Eps8 expression in cervical cancer samples was significantly higher compared with that in NC, LSIL and HSIL samples; thus, indicating the involvement of Eps8 in the development of cervical cancer. The analysis with regard to its clinical significance revealed that high Eps8 expression correlated with histological grade and lymph node metastases, suggesting that Eps8 serves a pivotal role in cervical cancer progression, and may be an independent biomarker for an unfavorable clinical prognosis. This is in agreement with previous observations that Eps 8 overexpression is associated with poor prognosis in patients with oral squamous cell carcinoma, mixed lineage leukaemia and ovarian cancer (33-35). It is generally accepted that decreased expression of E-cadherin, coupled with increased expression of vimentin, is characteristic of EMT (36). Therefore, the present study analyzed the possible association between the expression levels of Eps8 and EMT-associated proteins (E-cadherin and vimentin) in cervical carcinoma tissues. Correlation analysis revealed that high Eps8 expression was negatively correlated with the expression of E-cadherin and positively correlated with the expression of vimentin, implying the involvement of Eps8 in the EMT phenotype. Taken together, it was hypothesized that Eps8 makes a vital contribution to the progression of cervical cancer, partly through its influence on the EMT.

Given the results of the immunohistochemical analysis, the present study further investigated the role of Eps8 in the process of the EMT using shRNA-mediated knockdown of Eps8 in HeLa and SiHa cells. Eps8 knockdown appeared 
to cause a reversal of the EMT process in HeLa and $\mathrm{SiHa}$ cells, with changes observed in the expression of certain EMT markers (Fig. 2), including the E-cadherin upregulation and vimentin downregulation at both the mRNA and protein expression levels. E-cadherin is a major cell adhesion molecule involved in epithelial cell adhesion. Its loss or decreased expression has been suggested to be a key event in the EMT process. Reduced expression of E-cadherin serves an pivotal role in cancer progression and is associated with metastasis (37-42). E-cadherin is considered to be a prognostic biomarkers in certain solid malignant tumor types $(43,44)$. In addition, vimentin, as a canonical marker of the EMT, is widely expressed in mesenchymal cells. It has been previously reported that vimentin is overexpressed in various epithelial-derived tumor cells and tissues (45), and its overexpression is associated with enhanced tumor cell invasiveness (46). The present finding that Eps8 knockdown resulted in E-cadherin upregulation and vimentin downregulation suggested that Eps8 is involved in the EMT in HeLa and SiHa cells.

The present study also revealed that knockdown of Eps8 led to the downregulation of the transcription factor snail, which is hypothesized to be a key regulator of the EMT (47). The expression of snail has been implicated in suppressing the expression of E-cadherin and is involved in the initial migratory phenotype (48). Snail is expressed at the invasive front of tumors and is associated with tumor recurrence and lymph node metastasis, suggesting a role for snail in tumor progression (49). Other previous studies have indicated that Eps 8 activates AKT by stimulating phosphatidylinositol 4,5-bisphosphate 3-kinase (PI3K) (16), and that the PI3K/AKT-snail pathway is involved in the EMT in breast and lung cancer cells $(50,51)$. Furthermore, it was speculated that downregulation of snail by Eps8 silencing represents a plausible mechanism by which Eps8 is involved in the process of the EMT in cervical cancer cells. Furthermore, the present observation that Eps8 depletion in cell invasion and migration assays significantly impaired the invasive and migratory capacities of cervical cancer cells indicated that Eps8 serves a vital role in tumor progression.

In conclusion, the results of the present study demonstrated that the expression of Eps8 was significantly associated with the expression of EMT-related proteins in cervical cancer samples. Furthermore, these results indicated that Eps8 overexpression was correlated with lymphatic and distant metastasis of cervical cancer. Additionally, decreased expression of Eps8 impaired the EMT process and suppressed the migration and invasion of HeLa and SiHa cells. These results provided an insight into the important function of Eps8 in cervical cancer progression by orchestrating the EMT, and indicated that Eps8 is a novel potential target for developing therapeutic strategies for cervical cancer. However, the specific regulatory role of Eps8 in the EMT remains unknown and further in vitro and in vivo investigations are required to elucidate the detailed mechanism by which Eps8 regulates the EMT.

\section{Acknowledgements}

The authors would like to thank Dr Huijuan Zhang and Dr Bei Chen (Department of Pathology of the International Peace Maternity and Child Health Hospital of the China Welfare
Institute) for their assistance with immunohistochemistry. This research was supported by grants from the Project of Health and Family Planning Commission of Shanghai Municipality (no. 201440603), the National Natural Science Foundation of China (no. 81402134) and the Science and Technology Commission of Shanghai Municipality (no. 12ZR1451400).

\section{References}

1. Crosbie EJ, Einstein MH, Franceschi S and Kitchener HC: Human papillomavirus and cervical cancer. Lancet 382: 889-899, 2013.

2. Partridge EE, Abu-Rustum N, Giuliano A, Massad S, McClure J, Dwyer $M$ and Hughes $M$; National comprehensive cancer network: Cervical cancer screening. J Natl Compr Canc Netw 12: 333-341; quiz 341, 2014

3. Liu X, Wang D, Liu H, Feng Y, Zhu T, Zhang L, Zhu B and Zhang Y: Knockdown of astrocyte elevated gene-1 (AEG-1) in cervical cancer cells decreases their invasiveness, epithelial to mesenchymal transition, and chemoresistance. Cell Cycle 13: 1702-1707, 2014.

4. Kim CJ, Jeong JK, Park M, Park TS, Park TC, Namkoong SE and Park JS: HPV oligonucleotide microarray-based detection of HPV genotypes in cervical neoplastic lesions. Gynecol Oncol 89: 210-217, 2003.

5. Jung HY, Fattet L and Yang J: Molecular pathways: Linking tumor microenvironment to epithelial-mesenchymal transition in metastasis. Clin Cancer Res 21: 962-968, 2015.

6. Thiery JP, Acloque H, Huang RY and Nieto MA: Epithelial-mesenchymal transitions in development and disease. Cell 139: 871-890, 2009.

7. Kalluri R and Neilson EG: Epithelial-mesenchymal transition and its implications for fibrosis. J Clin Invest 112: 1776-1784, 2003

8. Fazioli F, Minichiello L, Matoska V, Castagnino P, Miki T, Wong WT and Di Fiore PP: Eps8, a substrate for the epidermal growth factor receptor kinase, enhances EGF-dependent mitogenic signals. EMBO J 12: 3799-3808, 1993.

9. Matoskova B, Wong WT, Salcini AE, Pelicci PG and Di Fiore PP: Constitutive phosphorylation of eps 8 in tumor cell lines: Relevance to malignant transformation. Mol Cell Biol 15: 3805-3812, 1995

10. Maa MC, Lee JC, Chen YJ, Chen YJ, Lee YC, Wang ST, Huang CC, Chow NH and Leu TH: Eps8 facilitates cellular growth and motility of colon cancer cells by increasing the expression and activity of focal adhesion kinase. J Biol Chem 282: 19399-19409, 2007.

11. Xu M, Shorts-Cary L, Knox AJ, Kleinsmidt-DeMasters B, Lillehei K and Wierman ME: Epidermal growth factor receptor pathway substrate 8 is overexpressed in human pituitary tumors: Role in proliferation and survival. Endocrinology 150: 2064-2071, 2009.

12. Yap LF, Jenei V, Robinson CM, Moutasim K, Benn TM, Threadgold SP, Lopes V, Wei W, Thomas GJ and Paterson IC: Upregulation of Eps8 in oral squamous cell carcinoma promotes cell migration and invasion through integrin-dependent Rac1 activation. Oncogene 28: 2524-2534, 2009.

13. Bashir M, Kirmani D, Bhat HF, Baba RA, Hamza R, Naqash S, Wani NA, Andrabi KI, Zargar MA and Khanday FA: P66shc and its downstream Eps8 and Rac1 proteins are upregulated in esophageal cancers. Cell Commun Signal 8: 13, 2010.

14. Welsch T, Endlich K, Giese T, Büchler MW and Schmidt J: Eps8 is increased in pancreatic cancer and required for dynamic actin-based cell protrusions and intercellular cytoskeletal organization. Cancer Lett 255: 205-218, 2007.

15. Liu PS, Jong TH, Maa MC and Leu TH: The interplay between Eps8 and IRSp53 contributes to Src-mediated transformation. Oncogene 29: 3977-3989, 2010.

16. Wang H, Patel V, Miyazaki H, Gutkind JS and Yeudall WA: Role for EPS8 in squamous carcinogenesis. Carcinogenesis 30: 165-174, 2009.

17. Chen YJ, Shen MR, Chen YJ, Maa MC and Leu TH: Eps8 decreases chemosensitivity and affects survival of cervical cancer patients. Mol Cancer Ther 7: 1376-1385, 2008.

18. Ackland ML, Newgreen DF, Fridman M, Waltham MC, Arvanitis A, Minichiello J, Price JT and Thompson EW: Epidermal growth factor-induced epithelio-mesenchymal transition in human breast carcinoma cells. Lab Invest 83: 435-448, 2003. 
19. Hagemann T, Bozanovic T, Hooper S, Ljubic A, Slettenaar VI, Wilson JL, Singh N, Gayther SA, Shepherd JH and Van Trappen PO: Molecular profiling of cervical cancer progression. Br J Cancer 96: 321-328, 2007.

20. Shen MR, Hsu YM, Hsu KF, Chen YF, Tang MJ and Chou CY: Insulin-like growth factor 1 is a potent stimulator of cervical cancer cell invasiveness and proliferation that is modulated by alphavbeta3 integrin signaling. Carcinogenesis 27: 962-971, 2006.

21. Adam L, Zhong M, Choi W, Qi W, Nicoloso M, Arora A, Calin G, Wang H, Siefker-Radtke A, McConkey D, et al: MiR-200 expression regulates epithelial-to-mesenchymal transition in bladder cancer cells and reverses resistance to epidermal growth factor receptor therapy. Clin Cancer Res 15: 5060-5072, 2009.

22. Cannito S, Novo E, di Bonzo LV, Busletta C, Colombatto S and Parola M: Epithelial-mesenchymal transition: From molecular mechanisms, redox regulation to implications in human health and disease. Antioxid Redox Signal 12: 1383-1430, 2010.

23. Wang Z, Li Y, Ahmad A, Azmi AS, Kong D, Banerjee S and Sarkar FH: Targeting miRNAs involved in cancer stem cell and EMT regulation: An emerging concept in overcoming drug resistance. Drug Resist Updat 13: 109-118, 2010.

24. Livak KJ and Schmittgen TD: Analysis of relative gene expression data using real-time quantitative PCR and the $2^{-\Delta \Delta C T}$ method. Methods 25: 402-408, 2001.

25. Qureshi R, Arora H and Rizvi MA: EMT in cervical cancer: Its role in tumour progression and response to therapy. Cancer Lett 356: 321-331, 2015.

26. Polyak K and Weinberg RA: Transitions between epithelial and mesenchymal states: Acquisition of malignant and stem cell traits. Nat Rev Cancer 9: 265-273, 2009.

27. Acloque H, Adams MS, Fishwick K, Bronner-Fraser M and Nieto MA: Epithelial-mesenchymal transitions: The importance of changing cell state in development and disease. J Clin Invest 119: 1438-1449, 2009.

28. Castagnino P, Biesova Z, Wong WT, Fazioli F, Gill GN and Di Fiore PP: Direct binding of eps8 to the juxtamembrane domain of EGFR is phosphotyrosine- and SH2-independent. Oncogene 10 : 723-729, 1995.

29. Disanza A, Mantoani S, Hertzog M, Gerboth S, Frittoli E, Steffen A, Berhoerster K, Kreienkamp HJ, Milanesi F, D Fiore PP, et al: Regulation of cell shape by Cdc42 is mediated by the synergic actin-bundling activity of the Eps8-IRSp53 complex. Nat Cell Biol 8: 1337-1347, 2006.

30. Goicoechea S, Arneman D, Disanza A, Garcia-Mata R, Scita G and Otey CA: Palladin binds to Eps8 and enhances the formation of dorsal ruffles and podosomes in vascular smooth muscle cells J Cell Sci 119: 3316-3324, 2006

31. Innocenti M, Frittoli E, Ponzanelli I, Falck JR, Brachmann SM, Di Fiore PP and Scita G: Phosphoinositide 3-kinase activates Rac by entering in a complex with Eps8, Abil, and Sos-1. J Cell Biol 160: 17-23, 2003

32. Wang H, Teh MT, Ji Y, Patel V, Firouzabadian S, Patel AA, Gutkind JS and Yeudall WA: EPS8 upregulates FOXM1 expression, enhancing cell growth and motility. Carcinogenesis 31: $1132-1141,2010$.

33. Chu PY, Liou JH, Lin YM, Chen CJ, Chen MK, Lin SH, Yeh CM, Wang HK, Maa MC, Leu TH, et al: Expression of Eps8 correlates with poor survival in oral squamous cell carcinoma. Asia Pac J Clin Oncol 8: e77-e81, 2012.

34. Kang H, Wilson CS, Harvey RC, Chen IM, Murphy MH, Atlas SR, Bedrick EJ, Devidas M, Carroll AJ, Robinson BW, et al: Gene expression profiles predictive of outcome and age in infant acute lymphoblastic leukemia: A children's oncology group study. Blood 119: 1872-1881, 2012.
35. Chen H, Wu X,PanZK and Huang S: Integrity of SOS1/EPS8/ABI1 tri-complex determines ovarian cancer metastasis. Cancer Res 70: 9979-9990, 2010.

36. Lim J and Thiery JP: Epithelial-mesenchymal transitions: Insights from development. Development 139: 3471-3486, 2012.

37. Schipper JH, Frixen UH, Behrens J, Unger A, Jahnke K and Birchmeier W: E-cadherin expression in squamous cell carcinomas of head and neck: Inverse correlation with tumor dedifferentiation and lymph node metastasis. Cancer Res 51: 6328-6337, 1991.

38. Shiozaki H, Tahara H, Oka H, Miyata M, Kobayashi K, Tamura S, Iihara K, Doki Y, Hirano S, Takeichi M, et al: Expression of immunoreactive E-cadherin adhesion molecules in human cancers. Am J Pathol 139: 17-23, 1991.

39. Kadowaki T, Shiozaki H, Inoue M, Tamura S, Oka H, Doki Y, Iihara K, Matsui S, Iwazawa T, Nagafuchi A, et al: E-cadherin and alpha-catenin expression in human esophageal cancer. Cancer Res 54: 291-296, 1994.

40. Bowie GL, Caslin AW, Roland NJ, Field JK, Jones AS and Kinsella AR: Expression of the cell-cell adhesion molecule E-cadherin in squamous cell carcinoma of the head and neck. Clin Otolaryngol Allied Sci 18: 196-201, 1993.

41. Koseki S, Aoki T, Ansai S, Hozumi Y, Mitsuhashi Y and Kondo S: An immunohistochemical study of E-cadherin expression in human squamous cell carcinoma of the skin: Relationship between decreased expression of E-cadherin in the primary lesion and regional lymph node metastasis. J Dermatol 26: 416-422, 1999 .

42. Wu H, Lotan R, Menter D, Lippman SM and Xu XC: Expression of E-cadherin is associated with squamous differentiation in squamous cell carcinomas. Anticancer Res 20: 1385-1390, 2000.

43. Berx G, Staes K, van Hengel J, Molemans F, Bussemakers MJ, van Bokhoven A and van Roy F: Cloning and characterization of the human invasion suppressor gene E-cadherin (CDH1). Genomics 26: 281-289, 1995.

44. Prognostic significance of CyclinD1 and E-Cadherin in patients with esophageal squamous cell carcinoma: Multiinstitutional retrospective analysis. Research committee on malignancy of esophageal cancer, Japanese society for esophageal diseases. J Am Coll Surg 192: 708-718, 2001.

45. Helfand BT, Mendez MG, Murthy SN, Shumaker DK, Grin B, Mahammad S, Aebi U, Wedig T, Wu YI, Hahn KM, et al: Vimentin organization modulates the formation of lamellipodia. Mol Biol CellMol Biol Cell 22: 1274-1289, 2011

46. Adam SA and Gerace L: Cytosolic proteins that specifically bind nuclear location signals are receptors for nuclear import. Cell 66: 837-847, 1991.

47. Moody SE, Perez D, Pan TC, Sarkisian CJ, Portocarrero CP, Sterner CJ, Notorfrancesco KL, Cardiff RD and Chodosh LA: The transcriptional repressor Snail promotes mammary tumor recurrence. Cancer Cell 8: 197-209, 2005.

48. Wu Y and Zhou BP: New insights of epithelial-mesenchymal transition in cancer metastasis. Acta Biochim Biophys Sin (Shanghai) 40: 643-650, 2008.

49. Guarino M: Epithelial-mesenchymal transition and tumour invasion. Int J Biochem Cell Biol 39: 2153-2160, 2007.

50. Chen KC, Chen CY, Lin CR, Yang TY, Chen TH, Wu LC and Wu CC: Luteolin attenuates TGF- $\beta 1$-induced epithelial-mesenchymal transition of lung cancer cells by interfering in the PI3K/Akt-NF-кB-Snail pathway. Life Sci 93: 924-933, 2013.

51. Zhang B, Yin C, Li H, Shi L, Liu N, Sun Y, Lu S, Liu Y, Sun L, Li X, et al: Nir1 promotes invasion of breast cancer cells by binding to chemokine (C-C motif) ligand 18 through the PI3K/Akt/GSK3 $\beta /$ Snail signalling pathway. Eur J Cancer 49: 3900-3913, 2013 